\title{
Four-Dimensional Characterization of the Babesia divergens Asexual Life Cycle, from the Trophozoite to the Multiparasite Stage
}

\author{
José Javier Conesa, a,b Elena Sevilla, c María Carmen Terrón, d Luis Miguel González,c Jeremy Gray, e Ana J. Pérez-Berná, ${ }^{\mathrm{b}}$ \\ José L. Carrascosa, ${ }^{a}$ Eva Pereiro, ${ }^{b}$ Francisco Javier Chichón, ${ }^{a}$ Daniel Luque, ${ }^{d}$ Estrella Monteroc \\ aDepartment of Structure of Macromolecules, Centro Nacional de Biotecnología, Consejo Superior de Investigaciones Científicas (CNB-CSIC), Madrid, Spain \\ bMistral Beamline, Experiment Division, ALBA Synchrotron (ALBA-CELLS), Barcelona, Spain \\ cParasitology Reference and Research Laboratory, Centro Nacional de Microbiología, Instituto de Salud Carlos III, Majadahonda, Madrid, Spain \\ dElectron and Confocal Microscopy Unit, Unidades Centrales Científico-Técnicas (SG-SAFI), Instituto de Salud Carlos III, Majadahonda, Madrid, Spain \\ eUCD School of Biology and Environmental Science, University College Dublin, Dublin, Ireland
}

José Javier Conesa and Elena Sevilla contributed equally to this article. Author order was determined on the basis of seniority.

ABSTRACT Babesia is an apicomplexan parasite of significance that causes the disease known as babesiosis in domestic and wild animals and in humans worldwide. Babesia infects vertebrate hosts and reproduces asexually by a form of binary fission within erythrocytes/red blood cells (RBCs), yielding a complex pleomorphic population of intraerythrocytic parasites. Seven of them, clearly visible in human RBCs infected with Babesia divergens, are considered the main forms and named single, double, and quadruple trophozoites, paired and double paired pyriforms, tetrad or Maltese Cross, and multiparasite stage. However, these main intraerythrocytic forms coexist with RBCs infected with transient parasite combinations of unclear origin and development. In fact, little is understood about how Babesia builds this complex population during its asexual life cycle. By combining cryo-soft X-ray tomography and video microscopy, main and transitory parasites were characterized in a native whole cellular context and at nanometric resolution. The architecture and kinetics of the parasite population was observed in detail and provide additional data to the previous $B$. divergens asexual life cycle model that was built on light microscopy. Importantly, the process of multiplication by binary fission, involving budding, was visualized in live parasites for the first time, revealing that fundamental changes in cell shape and continuous rounds of multiplication occur as the parasites go through their asexual multiplication cycle. A four-dimensional asexual life cycle model was built highlighting the origin of several transient morphological forms that, surprisingly, intersperse in a chronological order between one main stage and the next in the cycle.

IMPORTANCE Babesiosis is a disease caused by intraerythrocytic Babesia parasites, which possess many clinical features that are similar to those of malaria. This worldwide disease is increasing in frequency and geographical range and has a significant impact on human and animal health. Babesia divergens is one of the species responsible for human and cattle babesiosis causing death unless treated promptly. When $B$. divergens infects its vertebrate hosts, it reproduces asexually within red blood cells. During its asexual life cycle, $B$. divergens builds a population of numerous intraerythrocytic (IE) parasites of difficult interpretation. This complex population is largely unexplored, and we have therefore combined three- and four-dimensional imaging techniques to elucidate the origin, architecture, and kinetics of IE parasites. Unveiling the nature of these parasites has provided a vision of the $B$. divergens
Citation Conesa JJ, Sevilla E, Terrón MC González LM, Gray J, Pérez-Berná AJ, Carrascosa JL, Pereiro E, Chichón FJ, Luque D, Montero E. 2020. Four-dimensional characterization of the Babesia divergens asexual life cycle, from the trophozoite to the multiparasite stage. mSphere 5:e00928-20. https://doi.org/10.1128/ mSphere.00928-20.

Editor Ira J. Blader, University at Buffalo Copyright $\odot 2020$ Conesa et al. This is an open-access article distributed under the terms of the Creative Commons Attribution 4.0 International license.

Address correspondence to Daniel Luque, dluque@isciii.es, or Estrella Montero, estrella.montero@isciii.es.

Received 24 September 2020 Accepted 25 September 2020

Published 14 October 2020 
asexual cycle in unprecedented detail and is a key step to develop control strategies against babesiosis.

KEYWORDS Babesia divergens, cryo-soft X-ray tomography, intraerythrocytic asexual cycle, pathogen-host cell interactions, time-lapse video microscopy

abesia is an apicomplexan parasite which infect the red blood cells (RBCs) of a wide range of vertebrates, causing babesiosis (1). This disease, transmitted by ticks, has a significant impact on human and animal health. Two billion cattle worldwide are exposed to the infection causing substantial economic losses. The disease is also an emergent zoonosis of humans (2-4). Babesia divergens, is the most important species in Europe, causing redwater fever in cattle and severe and often fatal babesiosis in humans (1).

Once the vertebrate host has been bitten by an infected tick, sporozoites invade RBCs and begin an asexual life cycle known as merogony. This cycle has been partially elucidated and involves RBC invasion, metabolism and replication by a form of binary fission involving budding, resulting in merozoites that egress and destroy the host cell to seek and invade new uninfected RBCs (uRBCs) within seconds to minutes, thus perpetuating the infection $(5,6)$.

After several rounds of replication, $B$. divergens builds a complex population of distinct morphological intraerythrocytic (IE) stages. Seven of them, clearly distinguishable in the peripheral blood of infected humans and under in vitro growth conditions, are considered the main IE stages (7), namely, a single round trophozoite, paired pyriforms (two attached pear-shaped sister cells), double trophozoites (two round unattached cells), double paired pyriforms (two sets of paired sister cells), tetrads or Maltese Crosses (four attached sister cells), quadruple trophozoites (four round unattached cells), and multiple parasites (RBCs containing more than four parasites).

The merogony of Babesia is asynchronous and IE parasites in different stages coexist with free merozoites in the bloodstream (3). Despite the asynchronous nature of $B$. divergens replication, an approximation to the putative morphogenetic pathway was derived from in vitro methods alongside visible light microscopy, which allowed the visualization of a synchronized $B$. divergens asexual cycle for the first $24 \mathrm{~h}$, but exclusively involving the seven IE main stages (7). Sequentially, after the invasion of RBCs by free merozoites, the resulting single trophozoites give rise to paired pyriforms. Then, paired pyriforms develop into double trophozoites, which may give rise to double paired pyriforms. Paired pyriforms occasionally differentiate to tetrads or Maltese Crosses. Double paired pyriforms and tetrads result in quadruple trophozoites and, finally, quadruple trophozoites differentiate into multiple parasites (7). However, $24 \mathrm{~h}$ later, the life cycle progresses and lose its synchronicity, transforming into asynchronous populations in highly parasitized RBCs that contain transient morphological parasites of unclear origin alongside the seven main IE stages, mimicking the situation in human infections.

In spite of recent advances, our comprehension of the asexual $B$. divergens life cycle, including the multiplication process that a single parasite undergoes within an original infected RBC to develop ultimately into a multiparasite stage or the origin of tetrads, is still hampered by the limited knowledge of the kinetics and morphology of the parasite, currently based on information from light and electron microscopy of fixed and stained ultrathin sections (6-10). In this context, cryo-soft X-ray tomography (cryo-SXT) is a tool that bridges the gap between light and electron microscopy resolving some challenges in imaging and making unnecessary the use of contrasting agents, thus avoiding sectioning and staining artifacts (11-13). Taking into account these advantages, we use cryo-SXT here to obtain the three-dimensional (3D) reconstructions of cryopreserved, intact (nonsectioned) unstained $B$. divergens-infected RBCs (iRBCs) under close-to-native-state conditions. These data reveal not only the 3D architecture of the known main seven IE stages in their native environment but also 
novel transient IE parasites from $B$. divergens asynchronous cultures in a whole-cell context and at a nanometric resolution.

In addition to a detailed morphological description of $B$. divergens using cryo-SXT, our study is complemented by video microscopy over time (four-dimensional [4D] imaging) and transmission electron microscopy (TEM), thus providing insights into the kinetics of the parasite that reinforce and clarify previous $B$. divergens asexual life cycle models $(7,14,15)$. During this dynamic and pleomorphic in vitro cycle, it is possible to observe how $B$. divergens induces several cytological events that explain the origin and development of the main IE stages, as well as the role of the transient morphological parasites that surprisingly intersperse between one main IE stage and the next one in the cycle.

\section{RESULTS}

Three-dimensional structure of the $\boldsymbol{B}$. divergens blood stages. To characterize the 3D architecture of $B$. divergens, stained fluorescent parasites from in vitro asynchronous cultures were analyzed by correlative visible light fluorescence microscopy and cryo-SXT (Fig. 1). Acquisition of more than 200 cryo-SXT data sets were required to deal with the variety and complexity of the $B$. divergens pleomorphic forms involved throughout the parasite asexual life cycle.

Reconstructed tomograms were used to recover the intracellular 3D cartography of the main seven IE stages (Fig. 1 and 2). Three-dimensional data showed a morphological shape characteristic of each parasite stage. Thus, free merozoites are polarized ellipsoidal cells with an apical width prominence at the end (Fig. 2a and b). Single (Fig. 2c and d), double (Fig. 2e and f), and quadruple (Fig. $2 g$ and h) trophozoites showed a round shape, while paired pyriforms (Fig. $2 \mathrm{i}$ and j), tetrads (Fig. 2k and I), and double paired pyriforms (Fig. $2 \mathrm{~m}$ to $\mathrm{p}$ ) exhibited the characteristic pear-shaped form. Some 3D subcellular compartments were clearly discernible in both free merozoites and IE stages, including an elongated mitochondrion (1 to $2 \mu \mathrm{m}$ ) and a round apicoplast $(300 \mathrm{~nm})$ next to the nucleus $(700 \mathrm{~nm})$, which occupied most of the parasite cytoplasm. Dense granules were positioned on one side of the round trophozoites or close to the apical end of free merozoites and pear-shaped parasites (Fig. 2). It was not possible to resolve clearly the 3D structure of the Golgi apparatus and the endoplasmic reticulum (ER) due to the resolution attained ( $\sim 50 \mathrm{~nm}$ in $3 D)$. We also detected by cryo-SXT other intracellular structures in free merozoites and IE stages that may correspond to micronemes, rhoptries, and the inner membrane complex, previously observed by TEM $(8,9)$. However, it was not possible to unequivocally identify and/or count them (see Fig. $\mathrm{S} 1$ in the supplemental material).

Other membranous systems were clearly visible in the iRBCs. These include (i) possible hemoglobin-containing vesicles within the parasite cytoplasm and (ii) lowabsorbing vesicles with submicron size and novel long membrane structures, both within the cytoplasm of iRBCs. Thus, a single round dense feature was detected exclusively in the cytoplasm of trophozoites (Fig. S2a and b). Since these dense structures exhibited a similar X-ray linear absorption coefficient to the one of hemoglobin from the RBC cytoplasm, we hypothesized that they could be hemoglobincontaining vesicles. It is interesting to note that similar hemoglobin inclusions, and the possible parasite endocytic uptake of the hemoglobin from the cytoplasm of iRBCs, were observed in TEM serial sections (Fig. S2c to h). These membranous structures were heterogeneous in size ( 250 to $600 \mathrm{~nm}$ ) and could be the result of the internalization of an RBC cytoplasm portion to form the hemoglobin-containing vesicle.

The submicron vesicles present in the cytoplasm of iRBCs showed different sizes $(120,250$, and $400 \mathrm{~nm})$, and some of them were also observable by cryo-SXT and TEM (see Fig. S3).

Long structures $(1.5$ to $3 \mu \mathrm{m})$ appeared as a unique membranous extension. This feature extended from the parasite plasma membrane to the RBC plasma membrane, establishing a connection between the parasite and the RBC (see Fig. S4). 
a
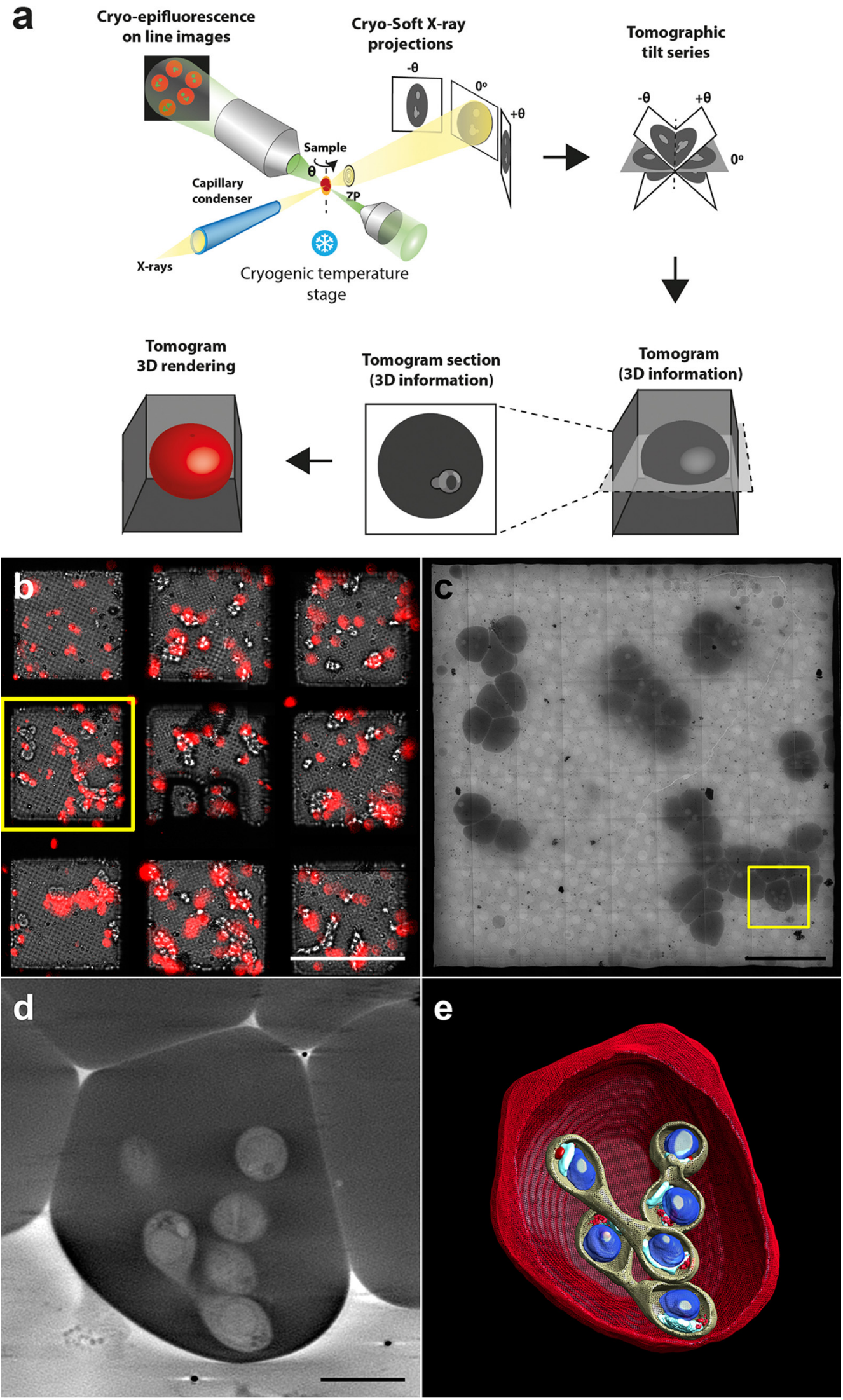

FIG 1 Correlative cryo-epifluorescence and cryo-soft X-ray tomography imaging of $B$. divergens human iRBCs. (a) This correlative workflow, used at MISTRAL beamline at ALBA synchrotron, provides biological images and structural information of (Continued on next page) 


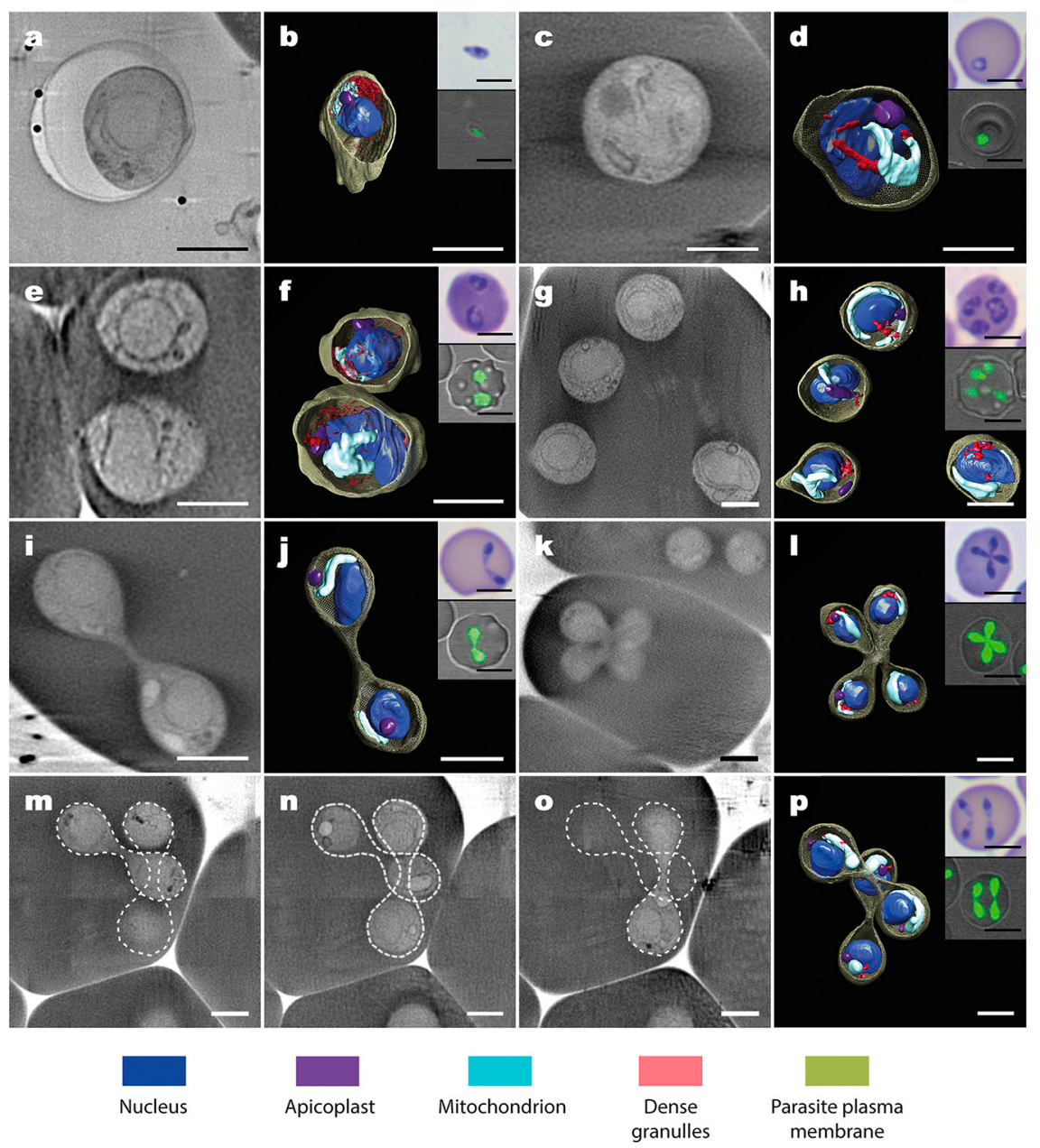

FIG 2 3D architecture of intact $B$. divergens parasites. Panels show the cryo-SXT tomogram sections of the $B$. divergens free merozoite and six main stages within human RBCs. The corresponding 3D tomogram renderings show the architecture of intact parasites and the organelle distribution in color. Insets show the equivalent parasite stages stained with Giemsa and observed by light microscopy (top inset) or stained with MitoTracker (green fluorescence) and observed using a confocal laser microscope (lower inset). (a and b) Free merozoite. ( $c$ and d) Single round trophozoite stage. (e and f) Double round trophozoite stage. ( $g$ and $h$ ) Quadruple round trophozoite stage. (i and j) Paired-pyriform stage. (k to l) Tetrad (Maltese Cross). ( $m$, n, and o) Tomogram sections of a double paired pyriform stage at different depths over the same 3D reconstruction. (p) 3D tomogram rendering of the corresponding double paired pyriform stage. The scale bars in panels a to $\mathrm{p}$ represent $2 \mu \mathrm{m}$. The scale bars in the insets in panels a to $\mathrm{p}$ represent $5 \mu \mathrm{m}$.

In addition to finding and recognizing the seven main IE stages in a whole-cell context, we observed other novel IE transient morphological forms with a complex pleomorphic 3D architecture. The elucidation of the origin and role of these new IE forms in the parasite life cycle was addressed using a combination of cryo-SXT and long-term time-lapse video microscopy, as described below.

FIG 1 Legend (Continued)

whole $B$. divergens human iRBCs close to their native state at a spatial resolution of around $50 \mathrm{~nm}$. B. divergens iRBCs are tilted to different angles, and an image is acquired at each angle. The tilt series of images are reconstructed into a 3D tomogram providing structural information of the whole cells. (b) B. divergens asynchronous in vitro cultures labeled with MitoTracker Red (red fluorescence) are deposited on to holey carbon EM grids in an optimal cell confluence $\left(10^{5}\right.$ cells per grid) and plunge-frozen in liquid ethane. The vitrified grids are screened with an online epifluorescence microscope to generate a fluorescence map and select the most relevant cells (yellow square). (c) Grids are loaded into the MISTRAL transmission X-ray microscope at the ALBA synchrotron light source for screening. An X-ray mosaic of projection images is generated and the previous fluorescence map helps in locating again the same cells in the yellow square. (d) Cryo-SXT tomogram sections of a $B$. divergens multiparasite stage acquired in the yellow squared area in panel c. (e) 3D rendering of the acquired cryo-SXT tomogram shown in panel $\mathrm{d}$. The scale bars in panels $\mathrm{b}, \mathrm{c}$, and $\mathrm{d}$ represent 100,20 , and $2 \mu \mathrm{m}$, respectively. 
Intraerythrocytic asexual cycle of $B$. divergens: from the trophozoite to the paired pyriform. Both asynchronous $B$. divergens in vitro culture and in peripheral blood of humans reflect a confused scenario of a heterogeneous parasite population when seen by standard microscopic techniques (Giemsa stain and light microscopy, as well as TEM). To define a comprehensive and chronological organization of these IE forms in the cycle, beginning with the single trophozoite development after RBC invasion and ultimately ending with the multiparasite stage formation, we filmed the asynchronous $B$. divergens culture for long periods and combined video microscopy and cryo-SXT data.

We captured images of newly iRBCs and RBCs already parasitized with single trophozoites. Video microscopy showed how these single trophozoites reproduced by a form of transverse binary fission that involves budding. Some details were also observable by cryo-SXT. In a first phase of development, trophozoites adopted amoeboid shapes (Fig. 3a and $2 \mathrm{~g}$ ) until they reached a round form with two protuberant buds (Fig. $3 \mathrm{~b}$ and $2 \mathrm{~h}$ ). This form was previously observed by TEM in B. divergens and more recently in B. bigemina and was named the budding form ("Mickey Mouse") because the buds contained organelles, indicating the posterior development of potential merozoites $(9,10)$. Video microscopy showed, step by step, how the budding form preceded the ultimate paired pyriform development in a second phase as was previously proposed (7). During this second phase, the budding form underwent a large change in morphology and became elongated; meanwhile, a transverse constriction was formed around the middle of the body (Fig. $2 \mathrm{i}$ and $3 \mathrm{c}$ ). The initial transverse constriction progressively changed to a protuberant knob that ultimately developed a narrow waist (Fig. $2 \mathrm{j}$ and $3 \mathrm{~d}$; see also Fig. S5). This fine structure divided the main body into two attached pear-shaped sister cells of equal size forming the paired pyriform stage (see Movie S1 at https://figshare.com/s/8ba6afd9e161899d682c). According to the cryo-SXT and TEM data, there are organelles and subcellular structures located in the zone that connect both sister cells. It seems that the development of the transverse constriction (Fig. $2 \mathrm{i}$ and $3 \mathrm{c}$ ) and the distribution of cell material contained inside (Fig. 3n; see also Fig. S5) occurred as a coordinated process resulting in the two identical attached cells, each of them with a complete set of organelles at the end of the process (Fig. 3o). Cryo-SXT 3D reconstructions also allowed us to visualize how trophozoite cartography changes in order to yield a paired pyriform (Fig. 3 I to o).

Notably, after imaging the morphogenesis of the trophozoite during its development by video microscopy and cryo-SXT, we were able to correctly identify and sequentially organize the corresponding IE forms when we saw them by light microscopy (Fig. 3q to t).

Moreover, by combining video microscopy and cryo-SXT, we obtained a 4D model description of the $B$. divergens asexual cycle starting with invasion by the free merozoite (Fig. 4a), followed by development of the single trophozoite into a paired pyriform within the human RBC (Fig. $4 b$ to $f$ and $j$ ).

The paired pyriform dominates the asexual cycle of $B$. divergens. After describing the dynamic development of the trophozoite, we continued analyzing the asexual cycle using the same experimental approach and following the chronological order established by (7). Hence, we captured images of RBCs parasitized with paired pyriforms entering into a dynamic cycle of multiple pathways. Instead of egressing from the host cell (6), an alternative development of the paired pyriform was to divide transversely yielding two pear-shaped cells. Several hours later, both cells adopted the characteristic round shape of the double trophozoite stage as was previously proposed (7). (Fig. 3e, f, j, k, o, and p and t to u, Fig. 4k to j; see also Fig. S6a in the supplemental material and Movie S2 at https://figshare.com/s/8ba6afd9e161899d682c). Our recent studies showed that the dissociation of single paired pyriforms into two pear-shaped sister cells occurs along the fine waist in a few seconds (6). However, the biomechanical process is not completely characterized and needs further study to understand the separation process. 


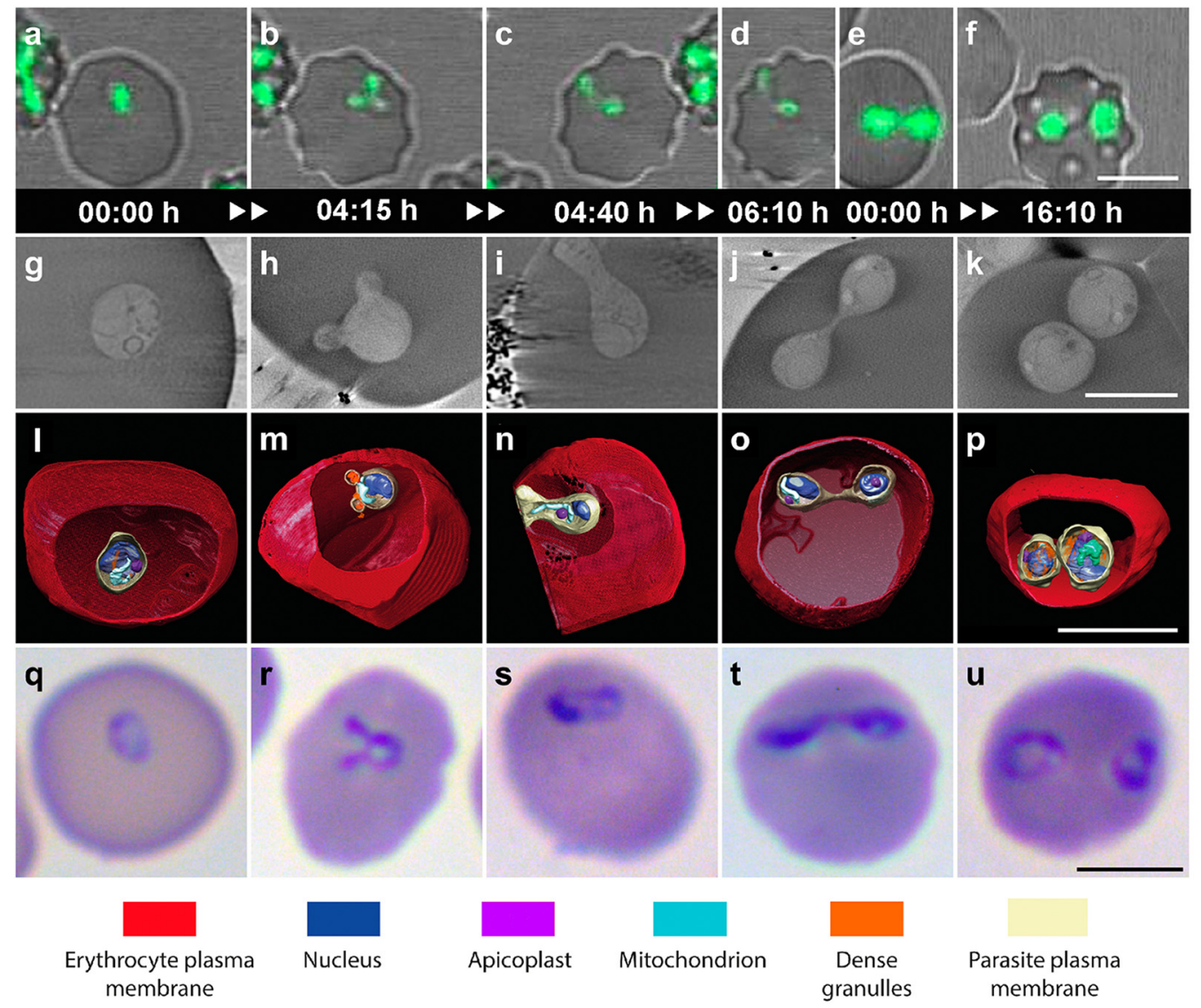

FIG 3 Dynamic development of single trophozoite and paired pyriform stages. The figure shows the development of the single trophozoite and paired pyriform stages and the transient forms that intersperse in a chronological order between both main stages within the human RBC. (a to $f$ ) Time-lapse image sequences, captured by video microscopy, of $B$. divergens parasites stained with MitoTracker (green fluorescence) within the human RBC. Equivalent IE parasite forms are identified in in vitro cultures by cryo-SXT and Giemsa stain and light microscopy. (g to k) Cryo-SXT tomogram sections of the $B$. divergens iRBCs. (I to p) The corresponding 3D tomogram renderings show the architecture of intact parasites and the organelle distribution in color. (q to $u$ ) $B$. divergens iRBCs stained with Giemsa. Panels are organized sequentially according to the video microscopy data. ( $a$ and b) A single trophozoite adopts amoeboid shapes until it reaches the budding form at $4 \mathrm{~h}$ and $15 \mathrm{~min}$. (c) The budding form develops into an early paired pyriform at $4 \mathrm{~h}$ and $40 \mathrm{~min}$. (d) The early paired pyriform ultimately develops into a paired pyriform stage at $6 \mathrm{~h}$ and $10 \mathrm{~min}$. (e and $\mathrm{f}$ ) The paired pyriform stage develops into the double trophozoite stage. $(\mathrm{g}, \mathrm{l}$, and $\mathrm{q}$ ) Single trophozoite stage. ( $\mathrm{h}, \mathrm{m}$, and $\mathrm{r}$ ) Budding form. (i, $\mathrm{n}$, and $\mathrm{s}$ ) Early paired pyriform under development. (j, o, and t) Paired-pyriform stage. (k, p, and u) Double trophozoite stage. (h to $\mathrm{m}$ ) Budding form showing the initial segregation of cell material that appears concentrate in both buds. (n) Detail of the transverse constriction of the main cellular body and the presence of some organelles across the constriction zone. (o) Each daughter pear-shaped cell inherits a complete set of organelles at the end of the binary fission. The scale bars in panels a to $\mathrm{k}$ and $\mathrm{q}$ to $\mathrm{u}$ represent $5 \mu \mathrm{m}$. The scale bars in panels $\mathrm{I}$ to $\mathrm{p}$ represent $2 \mu \mathrm{m}$. Time-lapse imaging was captured every $5 \mathrm{~min}$. The time lapse between each frame is indicated in hours and minutes (see also Movie S1 at https://figshare.com/s/8ba6afd9e161899d682c).

Continuing with the cycle reconstruction, double trophozoites were transformed into double paired pyriforms (7). During this dynamic process (Fig. 4k to o), each trophozoite multiplied by binary fission following the morphogenesis described above for a single trophozoite, but not necessarily simultaneously (see Fig. 56b and Fig. S7 in the supplemental material; see Movies S3 and S4 at https://figshare.com/ s/8ba6afd9e161899d682c).

Of considerable interest was the development of paired pyriforms into tetrads. Instead of dividing transversely, both sister pear-shaped cells remained attached and multiplied simultaneously yielding an attached double budding form or double "Mickey Mouse" that ultimately developed into a tetrad (Fig. 4e to g; see also Fig. S8a in the supplemental material and Movie S5 at https://figshare.com/s/ 8ba6afd9e161899d682c). We also observed that tetrads can separate, yielding double 


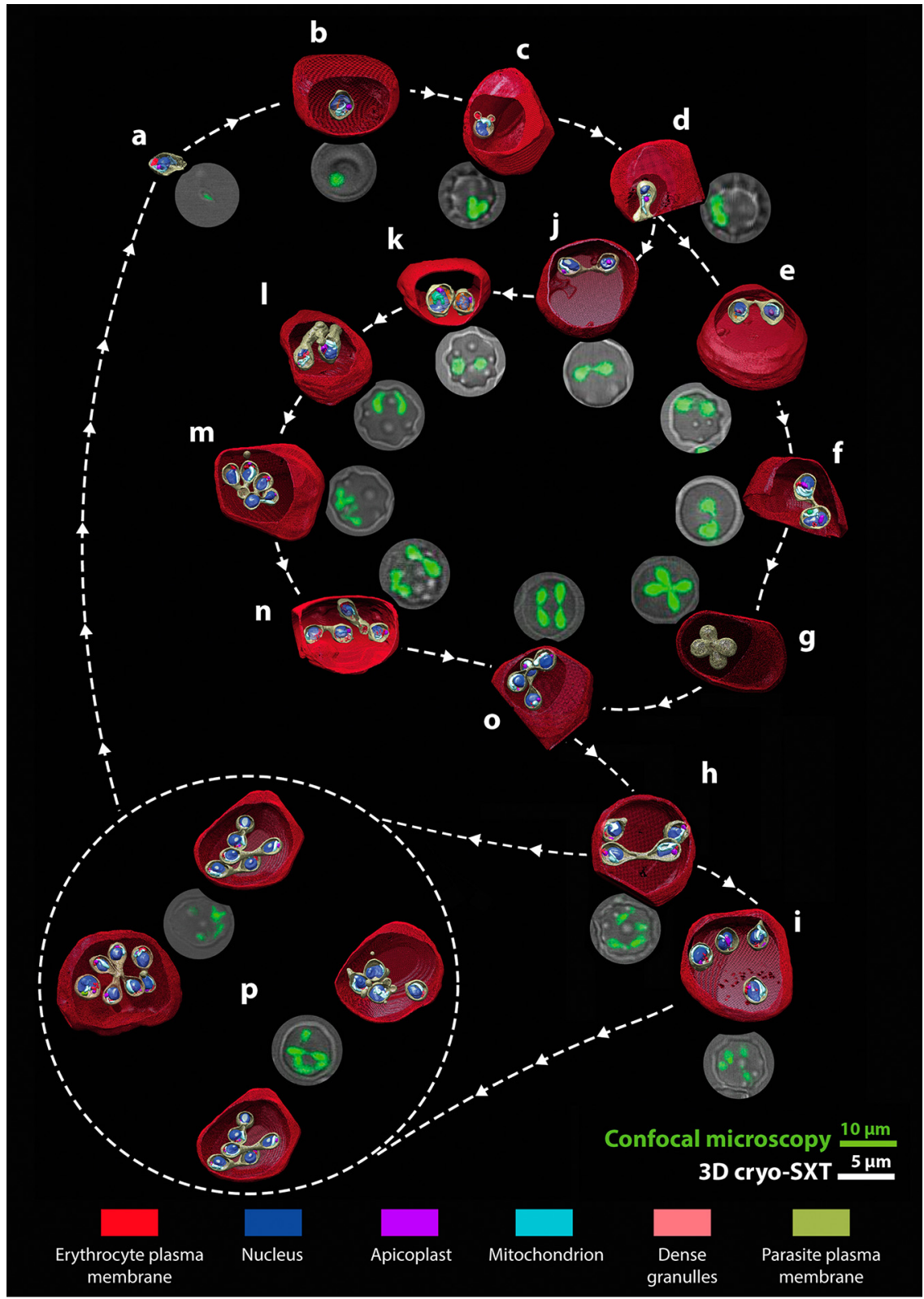

FIG 4 4D reconstruction of the main and intermediate IE stages that encompass the $B$. divergens asexual cycle. The cycle model shows a detailed chronological development that main IE stages undergo within the human RBC after the free merozoite invasion. (a to p) 3D rendering of cryo-SXT tomograms organized according to the time-lapse images generated for parasites at different stages of development (green fluorescence). Cellular compartments and the distribution of the parasite organelles are indicated in color. (a) A free merozoite about to invade a new human RBC. (b) Single trophozoite (main stage). (c) Budding form. (d) Early paired pyriform under development. (e) Paired-pyriform (main stage). (f) Initial development process of a paired pyriform into a tetrad. (g) Tetrad (main stage). (h) Two unattached pear-shaped parasites and a paired pyriform. (i) Quadruple trophozoites (main stage). (j) Paired pyriform (main stage). (k) Double trophozoites (main stage). (I) Two trophozoites adopting amoeboid forms during their development into double paired pyriforms. $(\mathrm{m})$ Double budding form. ( $\mathrm{n}$ and o) Double paired pyriforms (main stage). (p) Multiparasite stages (main stage). IE, intraerythrocytic.

paired pyriforms (Fig. $4 \mathrm{~g}$ and o; see also Fig. S8b and S9a in the supplemental material and Movies S6 and S7 at https://figshare.com/s/8ba6afd9e161899d682c), but we did not detect tetrads developing into quadruple trophozoites, as previously suggested (7).

Less frequently, the development of double paired pyriforms was observed to develop into quadruple trophozoites (Fig. 40 and h to i; see also Movie S8 at https:// figshare.com/s/8ba6afd9e161899d682c). 
TABLE 1 The time that intraerythrocytic parasites take to transform to the next stages ${ }^{a}$

\begin{tabular}{|c|c|}
\hline Intraerythrocytic-stage development & Mean $\pm \mathrm{SD}^{b}$ \\
\hline \multicolumn{2}{|l|}{ Trophozoite to paired pyriform $(n=14)$} \\
\hline Trophozoite to budding form & $3 \mathrm{~h} 33 \mathrm{~min} \pm 2 \mathrm{~h}$ \\
\hline Budding form to pyriform & $1 \mathrm{~h} 58 \mathrm{~min} \pm 51 \mathrm{~min}^{*}$ \\
\hline Total time & $5 \mathrm{~h} 31 \mathrm{~min} \pm 1 \mathrm{~h} 24 \mathrm{~min}$ \\
\hline \multicolumn{2}{|l|}{ Paired pyriforms to double trophozoites $(n=3)^{c}$} \\
\hline Total time & $7 \mathrm{~h} 37 \mathrm{~min} \pm 3 \mathrm{~h} 1 \mathrm{~min}$ \\
\hline \multicolumn{2}{|c|}{ Double trophozoites to double paired pyriforms $(n=7)$} \\
\hline First trophozoite to budding form & $6 \mathrm{~h} 38 \mathrm{~min} \pm 3 \mathrm{~h} 55 \mathrm{~min}$ \\
\hline Budding form to first pyriform & $1 \mathrm{~h} 58 \mathrm{~min} \pm 55 \mathrm{~min}^{*}$ \\
\hline Second trophozoite to budding form & $7 \mathrm{~h} 35 \mathrm{~min} \pm 5 \mathrm{~h} 4 \mathrm{~min}$ \\
\hline Budding form to second pyriform & $1 \mathrm{~h} 57 \mathrm{~min} \pm 50 \mathrm{~min}^{*}$ \\
\hline Total time & $9 \mathrm{~h} 32 \mathrm{~min} \pm 5 \mathrm{~h} 3 \mathrm{~min}$ \\
\hline \multicolumn{2}{|l|}{ Paired pyriform to tetrad $(n=11)$} \\
\hline Paired pyriform to double budding form & $4 \mathrm{~h} 14 \mathrm{~min} \pm 2 \mathrm{~h} 36 \mathrm{~min}$ \\
\hline Double budding form to tetrad & $1 \mathrm{~h} 48 \mathrm{~min} \pm 38 \mathrm{~min}^{*}$ \\
\hline Total time & $5 \mathrm{~h} 54 \mathrm{~min} \pm 2 \mathrm{~h} 40 \mathrm{~min}$ \\
\hline \multicolumn{2}{|l|}{ Tetrad to double paired pyriforms $(n=9)^{d}$} \\
\hline Total time & $3 \mathrm{~h} 3 \mathrm{~min} \pm 2$ h $54 \mathrm{~min}$ \\
\hline \multicolumn{2}{|c|}{ Double paired pyriforms to quadruple trophozoites $(n=1)^{e}$} \\
\hline Total time & 10 h $15 \mathrm{~min}$ \\
\hline \multicolumn{2}{|c|}{ Double paired pyriforms to multiparasite stage $(n=3)$} \\
\hline Total time & $7 \mathrm{~h} 40 \mathrm{~min} \pm 5 \mathrm{~h} 32 \mathrm{~min}$ \\
\hline \multicolumn{2}{|c|}{ Quadruple trophozoites to multiparasite stage $(n=1)$} \\
\hline Total time & $4 \mathrm{~h} 5 \mathrm{~min}$ \\
\hline \multicolumn{2}{|l|}{ Multiparasite stage development $(n=3)$} \\
\hline Total time & $5 \mathrm{~h} 20 \mathrm{~min} \pm 3 \mathrm{~h} 11 \mathrm{~min}$ \\
\hline
\end{tabular}

aThe table shows the average times that parasites take to transform from one main stage to the next. The phases of the development for some main stages are detailed in table footnotes. ${ }^{*}$, the time from the budding form or double budding form to the next stage is very similar between trophozoites. $n$, number of events recorded.

${ }^{b} \mathrm{SD}$, standard deviation.

cPaired pyriform splits into double trophozoites.

aTetrad splits into double paired pyriforms.

eDouble paired pyriforms split into quadruple trophozoites.

Double paired pyriforms and quadruple trophozoites are involved in the development of multiparasite $\boldsymbol{B}$. divergens stages. After formation of double paired pyriforms and quadruple trophozoites, the cycle continued with the development of multiparasite stages as was previously proposed (7) (Fig. 4h; see also Fig. S9b in the supplemental material and Movie S9 at https://figshare.com/s/8ba6afd9e161899d682c). Double paired pyriforms and quadruple trophozoites underwent consecutive rounds of multiplication following a complex pathway of development and resulting in different multiparasite stages or polyparasitized RBCs infected with multiple combinations of parasite forms (Fig. 4p; see also Fig. S10a in the supplemental material and Movie S10 at https://figshare.com/s/8ba6afd9e161899d682c). Notably, multiparasite stages underwent new rounds of multiplication before ultimately egressing from the host cell (see Fig. S10b in the supplemental material and Movie S11 at https://figshare.com/s/ 8ba6afd9e161899d682c).

Finally, the time IE parasites took to transform into the next stages was measured and evaluated (Table 1). Of special interest is the finding that, regardless of the stage and the asynchronous multiplication of two or more parasites within the same RBC, the time elapsed from the budding form to the resulting new stage was similar. That is, trophozoites from any stage took similar times from the budding form to the paired 
pyriform ( $1 \mathrm{~h} 38 \mathrm{~min} \pm 48 \mathrm{~min}$ ). This time was also comparable to the time required for the paired pyriforms when developed through a synchronized budding to tetrads (Table 1).

\section{DISCUSSION}

By combining cryo-SXT and video microscopy we have obtained reconstructions and data in unprecedented detail, which significantly clarifies our understanding of the asexual cycle of $B$. divergens. The correlation between both techniques provides a 4D vision of the cycle of native, live $B$. divergens parasites improving our concept of parasite development in the life cycle.

The cryo-SXT tomograms revealed the main IE stages, as well as unexpected forms of the parasite, that were also observed and recognized by video microscopy as intermediate IE forms. These intermediate forms that interposed in a sequential order between one main stage and the next explain the origin and development of trophozoites, pyriforms, tetrads, and multiparasite stages (see Movies S12 to S14 at https:// figshare.com/s/8ba6afd9e161899d682c).

Interestingly, the pattern of $B$. divergens population formation that we observed here is similar to that found in blood smears of infected humans rather than infected cattle. Thus, tetrads are not typically found in cattle but are common in human RBCs, and polyparasitism is also a side effect of the cycle that occurs in terminal clinical cases $(6,7,16,17)$.

The combination of video microscopy and cryo-SXT allowed the chronological ordering of a pattern of formation of the entire IE population, adopted by $B$. divergens, in the asexual cycle (Fig. 4). The proposed cycle model showed a complex morphological process where, for an individual trophozoite, there are several development options before exiting the RBC as was previously proposed (7). This phenomenon occurs when parasites, instead of egressing as free merozoites to invade new RBCs, undergo several rounds of multiplication, by binary fission involving budding, within the original iRBC (Fig. 4). This phenomenon ultimately gives rise to a diverse population of multiparasite stages (Fig. 4; see also Movie S14 at https://figshare.com/ s/8ba6afd9e161899d682c). Our results definitely indicated that multiparasite stages initially originated from a single trophozoite, confirming that the polyparasitism phenomenon is due to continuous rounds of multiplication $(17,18)$ rather than to multiple infections of the same RBC.

In the first phase of this process, the single trophozoite develops into a paired pyriform rather than undergoing a duplicate binary fission event to develop into a tetrad, as previously suggested (14). The new paired pyriform precedes all the next stages that may occur in the second phase of the asexual cycle, and the resulting paired pyriform can egress from the RBC (6) or remains within the cell to develop into a tetrad or a double trophozoite (7). Interestingly, the tetrad exclusively derives from the paired-pyriform stage. The tetrad development occurs when the paired pyriform, for unknown reasons, does not complete the fission process to separate in two trophozoites. As a consequence, the two pear-shaped forms that encompass the paired pyriform remain attached to each other, while both undergo a simultaneous but independent multiplication round involving budding. Each pear-shaped form yields two daughter cells, i.e., four attached cells in total, that form a tetrad. This could be a common cell biological feature of the Babesia spp. that, like $B$. divergens, are capable of producing tetrads $(19,20)$. Interestingly, attached or unattached, $B$. divergens parasites can just produce two daughter cells per parasite and per multiplication round.

The resulting tetrad, in contrast to other models (7), can egress or become double paired pyriforms within the RBC rather than develop to quadruple trophozoites. Double trophozoites, in contrast to other models (7), do not leave the cell but develop into double paired pyriforms. The latter may exit or remain within the original RBC and develop into quadruple trophozoites, a stage that does not leave the cell as well (6) but develops into a multiparasite stage (7). Moreover, double paired pyriforms undergo a 


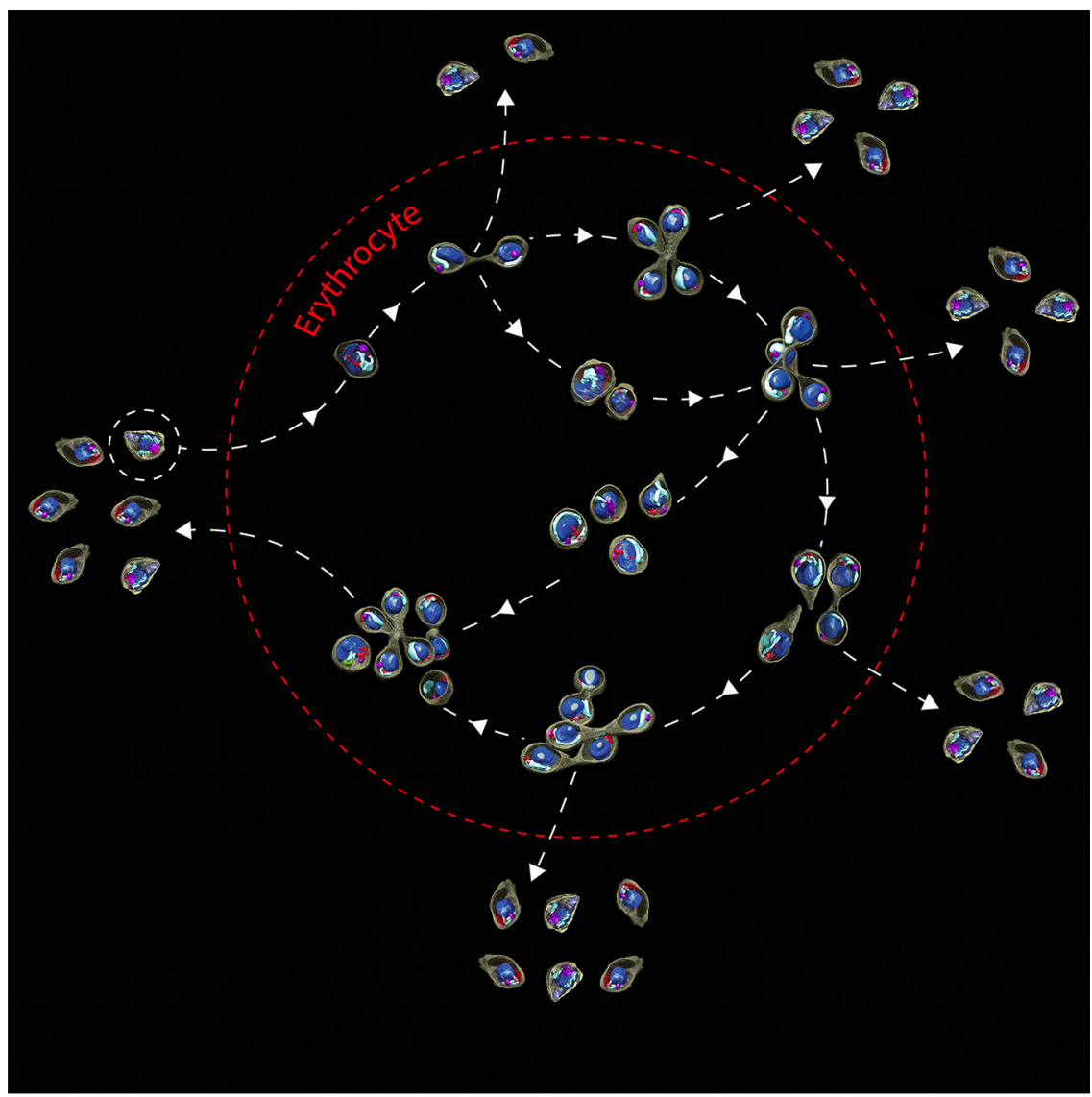

FIG 5 Simplified model of the $B$. divergens asexual cycle: from the single trophozoite to the multiparasite stage. The virtual model shows the transformation that a single trophozoite undergoes to become a multiparasite stage within one human RBC (red dotted line). This is possible through several rounds of multiplication by binary fission involving budding within the same host cell. This process starts with the invasion by a free merozoite (surrounded by a white dotted line) and its transformation into a single trophozoite inside the RBC. The single trophozoite develops into a paired pyriform. This new stage develops into double trophozoites or tetrads that precede double paired pyriforms. This last one may develop into quadruple trophozoites and/or multiparasite stages. Quadruple trophozoites can also develop into multiparasite stages. Importantly, paired pyriforms, double paired pyriforms, tetrads, and multiparasite stages, instead of developing within the RBC, can exit the host cell as free merozoites capable of invading new $\mathrm{RBC}$ s resulting in a rise in parasitemia.

novel pathway, not described previously, consisting of sequential rounds of multiplication to yield multiparasite stages without developing first into intermediate quadruple trophozoites. Finally, multiparasite stages egress from the host cell, and the resulting free merozoites invade new RBCs (Fig. 5).

Notably, the asexual cycle is an asynchronous process, and replication of two or more parasites within the same RBC does not necessarily occur simultaneously. Except for the paired pyriforms that develop into tetrads, we observed that most of the parasites multiply at different times in the presence of other parasites within the same host cell. These phenomena, far from occurring as random events, probably follow coordinated paths, tightly controlled by the ratios of invasion, development, and egression based on environmental conditions $(7,21)$.

The main stages grow slowly and multiply, by binary fission involving budding, within the human RBC for several hours in order to achieve the next stage. The main IE forms resulting are highly active and with a great capacity for deformation and transformation to intermediate or transitory forms during the process. In this context, the integration of cryo-SXT and video microscopy data also provided a comprehensive and dynamic view of the binary fission and budding process complementary to the static view provided previously by $\operatorname{TEM}(9,10)$. Indeed, it was possible to identify at least 
two key events to explain how fission occurs: (i) the formation of a local constriction and its transformation into a protuberant knob and ultimately into a narrow waist and (ii) the longitudinal stresses and the transverse rupture of the waist. While constriction formation is crucial to successful distribution of the cytoplasm to sister cells, narrow waist formation is essential for rupture to occur. Thus, a longitudinal tension force, exerted by the paired pyriform (6), probably increases longitudinal stresses in the waist to break this structure yielding two identical pear-shaped cells concluding the fission process (see Movie S14 at https://figshare.com/s/8ba6afd9e161899d682c). Fission has been recognized as a rapid process in such superior asexual organisms as freshwater planarians, which generate the forces necessary for division using only their own musculature and substrate traction (22). However, the fission process remains poorly understood in the majority of organisms because of the difficult of capturing it in real time (22). Taking into account that $B$. divergens encodes actin, actin polymerization and depolymerization proteins, and myosins A and B (23), it is possible that cytoskeleton components, as actin-myosin motors, play a role in parasite fission.

Apart from a detailed description of the 3D morphology and kinetics of $B$. divergens, our results provide, at the nanoscale, a cell cartography associated with cytological features and profound morphological changes undergone by $B$. divergens. These include the finding of trophozoites capable of internalizing host RBC hemoglobin by the formation of a local invagination of the parasite membrane and cytoplasm, resulting in a hemoglobin-containing vesicle within the parasite. This potential avidity for hemoglobin, apparently exclusively in round trophozoites, could presumably result in later hemoglobin catabolism and the production of nutrients to support $B$. divergens growth and replication during the life cycle. While this is a well-known strategy used by Plasmodium falciparum, it is poorly explored in Babesia spp. $(23,24)$. Recently, relevant orthologs of the $P$. falciparum papain proteases, involved in hemoglobin digestion, were identified in the $B$. divergens genome (23), shedding more light on the role of hemoglobin during the parasite life cycle.

After this stationary hemoglobin phase, trophozoites apparently do not egress from the host cell but grow and multiply by binary fission in order to provide pear-shaped parasites (Fig. 5). Then, these resulting paired pyriforms perpetuate the cycle by using two different strategies: (i) leaving the cell as free merozoites in order to invade new RBCs (6) or (ii) undergoing new rounds of multiplication in order to yield new IE trophozoites and pear-shaped parasites (7) (Fig. 5; see also Movies S13 and S14 at https://figshare.com/s/8ba6afd9e161899d682c).

During this dynamic cyclic process, $B$. divergens stages produce submicron vesicles observable within both the parasites and the iRBC cytoplasm (but not seen in $\mathrm{URBC}$ ), together with long membranous extensions connecting the IE parasite to the RBC plasma membrane. The presence and features of both membranous structures suggest the establishment of parasite/host cell interactions and the interchange of parasite/host cell products in a differ manner than the system of connected vesicles, used exclusively by $B$. microti and B. duncani iRBCs, for parasite antigen export (25).

Thus, both round trophozoites and pear-shaped forms are highly active and interacting parasites and have different but complementary roles. While trophozoites probably ensure the first nutrients by capturing hemoglobin, pear-shaped parasites seem to be the first step in perpetuating the cycle.

Undoubtedly, the life cycle of $B$. divergens requires precise strategies to ensure efficient propagation. Imaging tools showed a complex morphological presentation of main (7) but also novel transitory IE parasites and provide a better understanding of the role that $B$. divergens performs inside its host cell.

Further exploration of the whole Babesia life cycle, which spans two hosts-a tick vector and a vertebrate-will be crucial to improve our knowledge of the basic biology, morphology, and host-pathogen interactions of this parasite and to facilitate the parasite diagnosis and to provide better strategies for control. 


\section{MATERIALS AND METHODS}

Ethics statement. Human $A+$ blood from healthy donors was used to maintain cultures of $B$. divergens. The blood and protocol were approved for use by the Blood Transfusion Center, Madrid, Spain. Donors provided informed written consent for use of their blood for research purposes.

Parasite propagation. B. divergens asynchronous cultures (Bd Rouen 1987 strain) were maintained in vitro in human $A+R B C s$ at $5 \%$ hematocrit (9). Infected $R B C s$ were stained with Giemsa and examined with a Primo Star microscope (Zeiss, Germany) at $\times 100$ magnification.

Cryo-epifluorescence microscopy. Cultures of B. divergens at 30\% parasitemia were stained with MitoTracker Red FM mitochondrial stain (Thermo Fisher Scientific, Eugene, OR) at a final concentration of $500 \mathrm{nM}$ and according to the manufacturer's instructions. Then, $10^{5}$ fluorescence-stained cells were deposited on the surface of Au-G200F1 finder grids coated with holey carbon (R2/2; Quantifoil) and functionalized with poly-L-lysine (Merck, Germany) and fiducial gold markers (100 nm; BBI Solutions, UK) used for tomographic alignment purposes. To conserve the cellular structures and membrane arrangements in close-to-native conditions, cells attached to the grids were cryo-fixed by plunge freezing in liquid ethane using a Leica EM CPC plunge freezer (Leica Microsystems, Germany). Vitrified grids were transferred in liquid nitrogen to the cryo-correlative cooling stage (CMS196 stage; Linkam Scientific Instruments, UK) to hold samples at a stable $-190^{\circ} \mathrm{C}$ during analysis. The cryo-stage was inserted into an AxioScope A1 (Carl Zeiss, Germany) epifluorescence microscope with an N-Achroplan $10 \times / 0.25 \mathrm{Ph} 1$ objective and imaged with a CCD AxioCam ICm1 (Carl Zeiss). Cryo-fluorescence correlative microscopy was used to preselect vitrified samples and map cell coordinates. Selected samples were then transferred to ALBA synchotron (Barcelona, Spain) at liquid nitrogen temperature.

Cryo-soft X-ray tomography. Holey carbon-coated (R 2/2; Quantifoil) Au-G200F1 grids were analyzed in cryo-conditions by MISTRAL microscope at ALBA synchrotron (13). RBCs infected with red fluorescence $B$. divergens parasites were visualized on-line with a transmitted visible light and epifluorescence microscope integrated within the Mistral Soft X-Ray microscope to remap cell coordinates and select the cryo-SXT acquisition areas. X-ray projection mosaics were acquired to evaluate sample vitrification and thickness. Tilt series were acquired from $-65^{\circ}$ to $65^{\circ}$ at $1^{\circ}$ intervals, using $25-\mathrm{nm}$ zone plate lens objectives. The exposure time was 1 to $2 \mathrm{~s}$, depending on sample thickness, and an effective pixel size of $10 \mathrm{~nm}$. In addition, some samples were mounted in AutoGrid supports (FEl) and imaged following a dual-axis acquisition scheme. Most single-axis acquisition tomograms were done following a XTEND acquisition scheme (11). We imaged 218 acquisition areas as follows: 26 single-axis tilt series, 42 dual-axis tilt series, and 150 XTEND tilt series.

Tilt series were normalized to the flatfield, deconvolved by the measured apparent transfer function of the microscope (26) using python and MATLAB scripts and aligned with IMOD (27). XTEND data series were processed as described previously (28) using python scripts. Tomographic reconstructions were performed using TOMO3D software, 30 iterations of simultaneous iterative reconstruction technique (SIRT) algorithm (29) and edge enhanced using TOMOEED (30). Segmentation of volumes was carried out with SuRVoS (31), and volumes were represented with Chimera (32) and ImageJ (33).

Staining $B$. divergens culture parasites with MitoTracker green and subsequent treatment with concanavalin A. B. divergens cultures of at 25-28\% parasitemia were stained with MitoTracker Green FM mitochondrial stain (Thermo Fisher Scientific) at a final concentration of $500 \mathrm{nM}$ (6). Culture samples were placed in 6-well cell culture plates and maintained at $37^{\circ} \mathrm{C}$ in a humidified atmosphere of $5 \% \mathrm{CO}_{2}$ until use. Then, wells of a glass-bottom 96-Well Black (no. 1.5 coverslip, 5-mm glass diameter, uncoated; MatTek, Ashland, MA) were treated with $50 \mu$ l of concanavalin A (Sigma-Aldrich, St. Louis, MO) at $0.5 \mathrm{mg} / \mathrm{ml}$ for $10 \mathrm{~min}$ at room temperature and washed twice with $200 \mu \mathrm{l}$ of $1 \times$ PBS. Simultaneously, the $\mathrm{RBC}$ s infected with green fluorescence $B$. divergens parasites were also washed in $1 \times$ PBS. Cells $\left(5 \times 10^{5}\right.$ to $1 \times 10^{6}$ per well) were placed in the wells and stuck for $5 \mathrm{~min}$ at room temperature. Then, unbound cells were removed, and bound cells were washed twice with $200 \mu \mathrm{l}$ of $1 \times$ PBS. Finally, $1 \times$ PBS was replaced by $200 \mu \mathrm{l}$ of complete medium to maintain the culture during the video microscopy assays.

Long-term time-lapse recording and video processing. Time-lapse video was conducted using a Leica TCS SP5 confocal laser microscope (Leica Microsystems) equipped with epifluorescence microscopy (Leica DMI 6000B microscope) and incubation systems to control temperature, humidity, and $\mathrm{CO}_{2}$ conditions. To avoid loss of focus during the video recording, a 96-well plate containing RBCs infected with green fluorescence $B$. divergens parasites was placed under the confocal microscope with $63 \times$ oil objective lens and incubated in a $5 \% \mathrm{CO}_{2}$ environment at $37^{\circ} \mathrm{C}$ for $1 \mathrm{~h}$.

Time-lapse images of iRBCs were then recorded at one frame per 5 min interval using the following parameters: a 488-nm laser line and a laser level of $10 \%$, a speed of $700 \mathrm{~Hz}$, a $2.25 \mathrm{AU}$ pinhole aperture, a zoom of $2 \times, 2.5 \times$, or $3 \times$, and bright-field imaging under the same environmental conditions. Frames were captured for 18 to $21 \mathrm{~h}$ in a single z-section. The videos generated by the LAS AF software were processed with ImageJ and Fiji software $(33,34)$.

Transmission electron microscopy. For TEM ultrastructural analysis, $B$. divergens in vitro cultures were stuck to microscope cover glasses $(12 \mathrm{~mm})$ using poly-L-lysine (Merck). Briefly, samples were fixed in $2.5 \%$ glutaraldehyde and $2 \%$ paraformaldehyde in $0.1 \mathrm{M} \mathrm{Na}_{2} \mathrm{HPO}_{4}(\mathrm{pH} 7.4)$, postfixed with $1 \%$ osmium tetroxide and $1 \%$ uranyl acetate, dehydrated in increasing concentrations of ethanol, infiltrated using increasing concentrations of epoxy-resin, and polymerized at $60^{\circ} \mathrm{C}$ for $48 \mathrm{~h}$. Serial ultra- and semithin sections (70 to $150 \mathrm{~nm}$ ) were obtained with a Leica EM UC6 ultramicrotome and harvested according to standard procedures (8). Images were registered on a FEI Ceta camera with a Tecnai 12 FEI microscope operated at $120 \mathrm{kV}$.

Statistical analysis. Means and standard deviations (SD) were calculated using Excel 2010 (Microsoft, Redmond, WA), and results were expressed as averages \pm the SD. 
Data availability. Supplemental materials (Movies S1 to S14) are available at Figshare (https:// figshare.com/s/8ba6afd9e161899d682c).

\section{SUPPLEMENTAL MATERIAL}

Supplemental material is available online only.

FIG S1, PDF file, $0.8 \mathrm{MB}$.

FIG S2, PDF file, $2.6 \mathrm{MB}$.

FIG S3, PDF file, 1.1 MB.

FIG S4, PDF file, $1.3 \mathrm{MB}$.

FIG S5, PDF file, 1.5 MB.

FIG S6, PDF file, $2.4 \mathrm{MB}$.

FIG S7, PDF file, $1 \mathrm{MB}$.

FIG S8, PDF file, $2.2 \mathrm{MB}$.

FIG S9, PDF file, $1 \mathrm{MB}$.

FIG S10, PDF file, $2.1 \mathrm{MB}$.

\section{ACKNOWLEDGMENTS}

We thank the Centro de Transfusiones de la Comunidad de Madrid, which provided the human A + blood from healthy volunteer donors, and V. Lavilla for designing the animation movies.

This study was funded by grants from Ministerio de Economía y Competitividad from Spain (AGL2010-21774, AGL2014-56193-R to E.M. and L.M.G., and BFU201343149-R to D.L.). Cryo-SXT experiments were funded by ALBA synchrotron from Barcelona, Spain (proposals 2016021614 and 2017022084) and performed at MISTRAL beamline at ALBA Synchrotron with the collaboration of ALBA staff. E.S. was awarded a research fellowship from Plan Estatal de Investigación Científica y Técnica y de Innovación.

J.J.C., L.M.G., E.P., F.J.C., D.L., and E.M. designed research. J.J.C., E.S., M.C.T., L.M.G., J.G., A.J.P.-B., J.L.C., E.P., F.J.C., D.L., and E.M. conducted experiments and/or analysis. J.J.C., D.L., and E.M. wrote the paper. J.J.C. and E.S. made figures. J.J.C., E.S., M.C.T., L.M.G., A.J.P.-B., J.G., J.L.C., E.P., F.J.C., D.L., and E.M. conducted review and editing. L.M.G., D.L., E.P., and E.M. provided funding acquisition, project administration, and resources.

We declare there are no competing interests.

\section{REFERENCES}

1. Gray J, Zintl A, Hildebrandt A, Hunfeld K-P, Weiss L. 2010. Zoonotic babesiosis: overview of the disease and novel aspects of pathogen identity. Ticks Tick Borne Dis 1:3-10. https://doi.org/10.1016/j.ttbdis .2009.11.003.

2. Gohil S, Herrmann S, Günther S, Cooke BM. 2013. Bovine babesiosis in the 21st century: advances in biology and functional genomics. Int J Parasitol 43:125-132. https://doi.org/10.1016/j.ijpara.2012.09.008.

3. Jalovecka M, Hajdusek O, Sojka D, Kopacek P, Malandrin L. 2018. The complexity of piroplasms life cycles. Front Cell Infect Microbiol 8:248. https://doi.org/10.3389/fcimb.2018.00248.

4. Krause PJ. 2019. Human babesiosis. Int J Parasitol 49:165-174. https:// doi.org/10.1016/j.ijpara.2018.11.007.

5. Lobo CA, Rodriguez M, Cursino-Santos JR. 2012. Babesia and red cell invasion. Curr Opin Hematol 19:170-175. https://doi.org/10.1097/MOH .0b013e328352245a.

6. Sevilla E, González LM, Luque D, Gray J, Montero E. 2018. Kinetics of the invasion and egress processes of Babesia divergens, observed by timelapse video microscopy. Sci Rep 8:14116. https://doi.org/10.1038/s41598 $-018-32349-7$.

7. Cursino-Santos JR, Singh M, Pham P, Rodriguez M, Lobo CA. 2016. Babesia divergens builds a complex population structure composed of specific ratios of infected cells to ensure a prompt response to changing environmental conditions. Cell Microbiol 18:859-874. https://doi.org/10 $.1111 / \mathrm{cmi} .12555$.

8. Del Carmen Terrón M, González-Camacho F, González LM, Luque D, Montero E. 2016. Ultrastructure of the Babesia divergens free merozoite. Ticks Tick Borne Dis 7:1274-1279. https://doi.org/10.1016/j.ttbdis.2016.07.001.
9. Gorenflot A, Brasseur P, Precigout E, L'Hostis M, Marchand A, Schrevel J. 1991. Cytological and immunological responses to Babesia divergens in different hosts: ox, gerbil, man. Parasitol Res 77:3-12. https://doi.org/10 .1007/BF00934377.

10. Gubbels M-J, Keroack CD, Dangoudoubiyam S, Worliczek HL, Paul AS, Bauwens C, Elsworth B, Engelberg K, Howe DK, Coppens I, Duraisingh M. 2020. Fussing about fission: defining variety among mainstream and exotic apicomplexan cell division modes. Front Cell Infect Microbiol 10:269.

11. Chichón FJ, Rodríguez MJ, Pereiro E, Chiappi M, Perdiguero B, Guttmann P, Werner S, Rehbein S, Schneider G, Esteban M, Carrascosa JL. 2012. Cryo X-ray nano-tomography of vaccinia virus-infected cells. J Struct Biol 177:202-211. https://doi.org/10.1016/j.jsb.2011.12.001.

12. Pérez-Berná AJ, Rodríguez MJ, Chichón FJ, Friesland MF, Sorrentino A, Carrascosa JL, Pereiro E, Gastaminza P. 2016. Structural changes in cells imaged by soft X-ray cryo-tomography during hepatitis $\mathrm{C}$ virus infection. ACS Nano 10:6597-6611. https://doi.org/10.1021/acsnano.6b01374.

13. Sorrentino A, Nicolás J, Valcárcel R, Chichón FJ, Rosanes M, Avila J, Tkachuk A, Irwin J, Ferrer S, Pereiro E. 2015. MISTRAL: a transmission soft $\mathrm{X}$-ray microscopy beamline for cryo nano-tomography of biological samples and magnetic domains imaging. J Synchrotron Radiat 22:1112-1117. https://doi.org/10.1107/S1600577515008632.

14. Rossouw I, Maritz-Olivier C, Niemand J, van Biljon R, Smit A, Olivier NA, Birkholtz L-M. 2015. Morphological and molecular descriptors of the developmental cycle of Babesia divergens parasites in human erythrocytes. PLoS Negl Trop Dis 9:e0003711. https://doi.org/10.1371/journal .pntd.0003711. 
15. Valentin A, Rigomier D, Précigout E, Carcy B, Gorenflot A, Schrével J. 1991. Lipid trafficking between high density lipoproteins and Babesia divergens-infected human erythrocytes. Biol Cell 73:63-70. https://doi .org/10.1016/0248-4900(91)90010-k.

16. Asensi V, González LM, Fernández-Suárez J, Sevilla $E$, Navascués RÁ, Suárez ML, Lauret ME, Bernardo A, Carton JA, Montero E. 2018. A fatal case of Babesia divergens infection in Northwestern Spain. Ticks Tick Borne Dis 9:730-734. https://doi.org/10.1016/j.ttbdis.2018.02.018.

17. Zintl A, Mulcahy G, Skerrett HE, Taylor SM, Gray JS. 2003. Babesia divergens, a bovine blood parasite of veterinary and zoonotic importance. Clin Microbiol Rev 16:622-636. https://doi.org/10.1128/cmr.16.4 .622-636.2003.

18. Scudiero L, Mercado-Rojano WDJ, Rudolph A, Wang J, Laughery JM, Suarez CE. 2018. Comparisons of the topographic characteristics and electrical charge distributions among Babesia-infected erythrocytes and extraerythrocytic merozoites using AFM. J Microsc 271:84-97. https:// doi.org/10.1111/jmi.12697.

19. Conrad PA, Kjemtrup AM, Carreno RA, Thomford J, Wainwright K, Eberhard M, Quick R, Telford SR. 2006. Description of Babesia duncani n.sp. (Apicomplexa: Babesiidae) from humans and its differentiation from other piroplasms. Int J Parasitol 36:779-789. https://doi.org/10.1016/j ijpara.2006.03.008.

20. Rudzinska MA. 1981. Morphologic aspects of host-cell-parasite relationships in babesiosis, p 87-141. In Ristic M, Kreier JP (ed), Babesiosis. Academic Press, New York, NY.

21. Lobo CA, Cursino-Santos JR, Singh M, Rodriguez M. 2019. Babesia divergens: a drive to survive. Pathogens 8:95. https://doi.org/10.3390/ pathogens8030095.

22. Malinowski PT, Cochet-Escartin O, Kaj KJ, Ronan E, Groisman A, Diamond $\mathrm{PH}$, Collins E-MS. 2017. Mechanics dictate where and how freshwater planarians fission. Proc Natl Acad Sci U S A 114:10888-10893. https:// doi.org/10.1073/pnas.1700762114.

23. González LM, Estrada K, Grande R, Jiménez-Jacinto V, Vega-Alvarado L, Sevilla E, de la Barrera J, Cuesta I, Zaballos Á, Bautista JM, Lobo CA, Sánchez-Flores A, Montero E. 2019. Comparative and functional genomics of the protozoan parasite Babesia divergens highlighting the invasion and egress processes. PLoS Negl Trop Dis 13:e0007680. https://doi.org/ 10.1371/journal.pntd.0007680.

24. Brayton KA, Lau AOT, Herndon DR, Hannick L, Kappmeyer LS, Berens SJ, Bidwell SL, Brown WC, Crabtree J, Fadrosh D, Feldblum T, Forberger HA, Haas BJ, Howell JM, Khouri H, Koo H, Mann DJ, Norimine J, Paulsen IT, Radune D, Ren Q, Smith RK, Suarez CE, White O, Wortman JR, Knowles DP, McElwain TF, Nene VM. 2007. Genome sequence of Babesia bovis and comparative analysis of apicomplexan hemoprotozoa. PLoS Pathog 3-1413. https://doi.org/10.1371/journal.ppat.0030148.

25. Thekkiniath J, Kilian N, Lawres L, Gewirtz MA, Graham MM, Liu X, Ledizet M, Ben Mamoun C. 2019. Evidence for vesicle-mediated antigen export by the human pathogen Babesia microti. Life Sci Alliance 2:e201900382. https://doi.org/10.26508/lsa.201900382.

26. Otón J, Pereiro E, Pérez-Berná AJ, Millach L, Sorzano COS, Marabini R, Carazo JM. 2016. Characterization of transfer function, resolution and depth of field of a soft X-ray microscope applied to tomography enhancement by Wiener deconvolution. Biomed Opt Express 7:5092-5103. https://doi.org/10.1364/BOE.7.005092.

27. Mastronarde DN. 2008. Correction for non-perpendicularity of beam and tilt axis in tomographic reconstructions with the IMOD package. J Microsc 230:212-217. https://doi.org/10.1111/j.1365-2818.2008.01977.x.

28. Otón J, Pereiro E, Conesa JJ, Chichón FJ, Luque D, Rodríguez JM, Pérez-Berná AJ, Sorzano COS, Klukowska J, Herman GT, Vargas J, Marabini R, Carrascosa JL, Carazo JM. 2017. XTEND: extending the depth of field in cryo soft X-ray tomography. Sci Rep 7:45808. https://doi.org/10 .1038 /srep45808.

29. Agulleiro Jl, Fernandez JJ. 2011. Fast tomographic reconstruction on multicore computers. Bioinformatics 27:582-583. https://doi.org/10 $.1093 /$ bioinformatics/btq692.

30. Moreno JJ, Martínez-Sánchez A, Martínez JA, Garzón EM, Fernández JJ. 2018. TomoEED: fast edge-enhancing denoising of tomographic volumes. Bioinformatics 34:3776-3778. https://doi.org/10.1093/bioinformatics/ bty435.

31. Luengo I, Darrow MC, Spink MC, Sun Y, Dai W, He CY, Chiu W, Pridmore T, Ashton AW, Duke EMH, Basham M, French AP. 2017. SuRVoS: SuperRegion Volume Segmentation workbench. J Struct Biol 198:43-53. https://doi.org/10.1016/j.jsb.2017.02.007.

32. Pettersen EF, Goddard TD, Huang CC, Couch GS, Greenblatt DM, Meng EC, Ferrin TE. 2004. UCSF Chimera: a visualization system for exploratory research and analysis. J Comput Chem 25:1605-1612. https://doi.org/10 .1002/jcc.20084.

33. Schneider CA, Rasband WS, Eliceiri KW. 2012. NIH Image to ImageJ: 25 years of image analysis. Nat Methods 9:671-675. https://doi.org/10 .1038/nmeth.2089.

34. Schindelin J, Arganda-Carreras I, Frise E, Kaynig V, Longair M, Pietzsch T, Preibisch S, Rueden C, Saalfeld S, Schmid B, Tinevez J-Y, White DJ, Hartenstein V, Eliceiri K, Tomancak P, Cardona A. 2012. Fiji: an opensource platform for biological-image analysis. Nat Methods 9:676-682. https://doi.org/10.1038/nmeth.2019. 